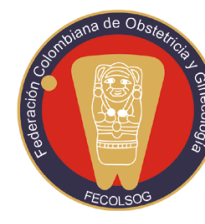

\title{
A PROPÓSITO DE LA “MISIÓN DE SABIOS”: ALGUNAS REFLEXIONES SOBRE INVERSIÓN EN CIENCIA Y TECNOLOGÍA EN EL SECTOR SALUD DEL PAÍS
}

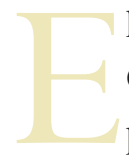

l actual Gobierno de Colombia, en cabeza de Colciencias y bajo la supervisión de la Vicepresidencia, convocó la llamada "Misión de Sabios” en febrero del presente año (1). Dicha Misión está conformada por ocho focos temáticos que trabajan en diferentes áreas del conocimiento o sectores económicos. Uno de los focos es el de Ciencias de la Salud y la Vida. Como tarea transversal a todos los grupos está el hacer recomendaciones respecto a cómo mejorar la educación como un primer paso para superar el atraso en ciencia y tecnología del país. Se parte del supuesto según el cual el desarrollo científico y tecnológico está orientado a solucionar los problemas del diario vivir, conduce a un desarrollo sostenible, incrementa el crecimiento económico y, por tanto, es un medio para mejorar las condiciones de vida del ser humano.

Quisiera hacer una reflexión sobre algunas posibles alternativas por considerar que podrían llevar en los próximos años al desarrollo de la investigación, la innovación y el desarrollo tecnológico en el sector salud y las ciencias de la vida, con un positivo efecto sobre el pueblo colombiano.

Según la Organización para la Cooperación y el Desarrollo Económicos (OCDE), la investigación y el desarrollo experimental comprenden el trabajo creativo y sistemático realizado con el objetivo de aumentar el volumen de conocimiento y concebir nuevas aplicaciones a partir del conocimiento disponible (2). La innovación, por su parte, se entiende como la implementación de un nuevo producto, servicio o proceso, o la mejora sustancial, o nuevos métodos de organización en el lugar de trabajo o en relaciones externas (3). Inicialmente, haré una aproximación a cuáles serían las condiciones de morbilidad que deberían ser objeto de investigación básica, aplicada o psicosocial, y cuáles deberían ser objeto de innovaciones de tipo tecnológico, educativo, organizacional o social, y luego abordaré otros criterios por considerar en la definición de prioridades.

Respecto a las condiciones de morbilidad, en el documento de la Organización Mundial de la Salud (OMS), la OCDE y la Organización Mundial de la Propiedad Intelectual (OMPI) sobre "Cómo promover el acceso a las tecnologías médicas y la innovación”, publicado en 2013, se describía que la carga de la morbilidad de los países de medianos ingresos, además de estar constituida por enfermedades crónicas no transmisibles, similar a la de los países de altos ingresos, tenía un importante componente dado por el VIH/Sida, la tuberculosis y los accidentes de tránsito, mientras que para los países de bajos ingresos la principal causa de morbilidad seguían siendo las enfermedades infecciosas (4).

Ahora revisemos la situación de Colombia, uno de los países más inequitativos de América según el Banco Mundial (5), situación que se refleja en las diferencias en la salud de sus regiones, algunas con una morbilidad e indicadores de salud con niveles similares a los países de altos ingresos, y otras regiones con niveles similares a las de países de África.

Por ejemplo, miremos algunos indicadores de mortalidad. En cuanto a la mortalidad materna (MM), para el año 2017 la razón de MM nacional fue de 
$50 \times 100.000$ nacidos vivos (NV) (6). Sin embargo, en departamentos como Chocó o La Guajira, la razón de MM es 4 a 5 veces mayor, mientras en Bogotá o en el Valle del Cauca es la mitad. Al mirar las causas de mortalidad materna, la preeclampsia sigue siendo la primera causa de muerte, seguida de las complicaciones del parto, posiblemente por hemorragia. En preeclampsia se requiere investigación básica aplicada y social ya que no se conoce su causa, ni se dispone de un tratamiento preventivo o curativo más allá de suspender el embarazo. En cuanto a la hemorragia, se requiere innovación en la organización del trabajo para ofrecer mejores servicios a las mujeres durante el parto.

Respecto a la mortalidad infantil, la tasa nacional para el 2017 fue de 10,7 x 1000 NV. Las principales causas son la mortalidad perinatal, asociada especialmente a la prematurez y el bajo peso al nacer y las malformaciones congénitas, posteriormente la infección respiratoria aguda y las enfermedades infecciosas y parasitarias (7). En la salud infantil se requiere investigación básica, aplicada y social sobre cómo prevenir el parto pretérmino, e innovación social, tecnológica y educativa respecto a cómo proveer y utilizar adecuadamente el agua potable para prevenir muchas de las infecciones mencionadas. Seguimos teniendo problemas con el dengue, la malaria y el zika (6). En todas las anteriores condiciones, que afectan también a los países de bajos ingresos, se observa que los más pobres, los que viven en la ruralidad, los afros y los indígenas son los más afectados.

Respecto a las condiciones crónicas, encontramos que las principales causas de muerte en Colombia son el infarto agudo de miocardio y la enfermedad cerebrovascular, seguidos por los homicidios, los accidentes de tránsito, la hipertensión arterial y la enfermedad pulmonar obstructiva crónica (EPOC) (8). Respecto al cáncer, la mayor incidencia se encuentra en el cáncer de próstata, de seno, colorrectal, de estómago y de cuello uterino, todas estas, patologías comunes a los países de altos ingresos (9).

¿Qué otros aspectos habría que considerar para definir las prioridades en investigación e innovación y desarrollo? Una sería la disponibilidad de recursos; en ese sentido, la biodiversidad que tiene Colombia debería ser considerada como un factor diferenciador a nivel global en la búsqueda de nuevos medicamentos. Otro factor por considerar son las nuevas tendencias en la medicina actual en los países de altos ingresos, orientadas al uso de la genómica, la terapia génica, la proteómica, la epigenética, los medicamentos biológicos que han cambiado drásticamente el curso clínico de algunas enfermedades como la hepatitis $\mathrm{C}$, algunos tipos de leucemia o enfermedades huérfanas, aunque, en muchos casos, a unos precios exorbitantes (10).

En el ejercicio de la medicina actual nos enfrentamos a dos grandes tendencias parcialmente opuestas: por un lado, la conservación y promoción de la salud y la prevención de la enfermedad y, por otro, la utilización de métodos diagnósticos y medicamentos más sofisticados orientados a la medicina personalizada. En la primera aproximación se encuentran quienes consideran que se requiere trabajar en la prevención del consumo de tabaco y alcohol, en los malos hábitos alimenticios y de vida, la inactividad y el deterioro del medio ambiente (4), las cuales son causa de muchas de las enfermedades crónicas no transmisibles, que para el 2030 serán la principal causa de morbilidad en todas las regiones del mundo, con una importante proporción de población en la tercera edad. Para lograr este objetivo por esta vía se requieren innovaciones en la educación en la primera infancia, época en que se da el mayor desarrollo cerebral, o en la adolescencia, para que nuestros niños y niñas aprendan hábitos alimentarios sanos y estilos de vida saludables y dispongan de un medio ambiente sano que tendrán un impacto importante en la prevención de la enfermedad cardiovascular, las enfermedades metabólicas, el EPOC y algunos de los tipos más frecuentes de cáncer. Otra opción sería investigar con nuestros recursos el desarrollo de nuevos medicamentos para las condiciones más prevalentes, o lograr la producción de biosimilares para reducir el precio de estos medicamentos como lo hacen en la India y Brasil.

Si tenemos que definir dónde ubicar los recursos de ciencia y tecnología en salud, podríamos sugerir que algunos deberían ir a la investigación de las condiciones 
que son propias de nuestra población, que tienen una gran carga social y en las cuales poco interés podrían tener los países de altos ingresos. Condiciones de salud como la preeclamsia, el dengue, la malaria, la enfermedad de Chagas y la leishmaniasis podrían recibir fondos para ser estudiadas desde el punto de vista de las ciencias básicas (genética, biología molecular, etc.) y la investigación aplicada en el desarrollo de vacunas o nuevos medicamentos a partir de nuestra diversidad biológica. Para esto se requiere que nuestros hospitales universitarios y los de enseñanza públicos tengan la capacidad de llevar a cabo la investigación clínica de los nuevos medicamentos por desarrollar en el país. Se requiere elevar los estándares de calidad y seguridad de la atención en nuestras instituciones públicas, otra área en la que se observa una importante inequidad en la salud en Colombia. Por otra parte, habrá que hacer investigación en ciencias sociales y neurociencias para evaluar cómo podemos reducir los altos niveles de violencia, embarazo en adolescentes o enfermedad mental en Colombia.

En relación con otras condiciones que nos afectan debemos invertir en proyectos de innovación en los procesos de acceso a los servicios básicos de salud (enfermedad diarreica aguda o infección respiratoria aguda en niños) o en procesos de atención (aborto seguro, cáncer). Por otra parte, la situación de envejecimiento nos debe conducir a hacer innovaciones en las llamadas tecnologías de asistencia que proveen servicios que incrementan la autonomía y el bienestar de los sujetos tales como prótesis, recordatorios, aplicaciones (apps) para atención domiciliaria a distancia, prendas inteligentes, etc. (11).

Deberemos definir si con la investigación y el desarrollo tecnológico los colombianos queremos construir un futuro en el cual podamos superar los factores que han determinado la pobreza y sus consecuencias para la salud, a fin de vivir en equidad, con bienestar, en armonía con nuestros congéneres y la naturaleza, para además morir dignamente como final natural de la vida, o si más bien decidimos que este desarrollo científico y tecnológico se oriente a la búsqueda de la inmortalidad o la eterna juventud.
Se ha planteado la selección de un proyecto emblemático para Colombia, el cual se deberá desarrollar en los próximos 20 años; este podría ser la reducción de la mortalidad infantil o la mortalidad materna a los niveles de los países de altos ingresos, o la disminución de la incidencia de la diabetes, la obesidad, la enfermedad hipertensiva, el cáncer o la enfermedad mental. Para esto nos apoyaríamos en las tecnologías convergentes, las ciencias básicas, las ciencias sociales, la cultura, la biotecnología, las cuales nos proveerían los medios para alcanzar el objetivo. Para esto requerimos un gran acuerdo entre todos los sectores de la sociedad y las áreas del conocimiento a fin de llegar a proyectos que nos unan y nos permitan vivir en paz y con bienestar.

\section{Hernando Gaitán Duarte, MD, MSc}

Editor

Profesor Titular

Universidad Nacional de Colombia

\section{REFERENCIAS}

1. Colciencias, Misión de Sabios Colombia; 2019. Disponible en: https://www.colciencias.gov.co/mision sabios

2. Organization for Economic Cooperation and Development (OECD). Frascati Manual 2015: Guidelines for Collecting and Reporting Data on Research and Experimental Development. The Measurement of Scientific, Technological and Innovation Activities. Paris: OECD; 2015. https:doi.org/10.1787/9789264239012-en

3. Organization for Economic Cooperation and Development (OECD). Oslo Manual: Guidelines for Collecting and Interpreting Innovation Data (3rd ed.). Paris: OECD; 2005.

4. Organización Mundial de la Salud (OMS), Organización Mundial de la Propiedad Intelectual (OMPI) y Organización Mundial del Comercio (OMC). Promover el acceso a las tecnologías médicas y la innovación. Intersecciones entre la salud pública, la propiedad intelectual y el comercio. Departamento de Salud Pública, Innovación y Propiedad Intelectual. Ginebra; 
2013. Disponible en: https:/www.wipo.int/edocs/ pubdocs/es/wipo_pub_628.pdf

5. Banco Mundial. Índice de Gini; 2019. Disponible en: https://datos.bancomundial.org/indicador/si.pov. gini?view $=$ map

6. Instituto Nacional de Salud(INS). Boletín Epidemiológico semana 52; 2017. Disponible en: https://www.ins.gov.co/ buscador-eventos/BoletinEpidemiologico/2017\%20 Bolet\%C3\%ADn\%20epidemiol\%C3\%B3gico\%20 semana\%2052.pdf

7. Ministerio de Salud y Protección Social. Análisis de Situación de Salud (ASIS), Colombia, 2018. Dirección de Epidemiología y Demografía. Bogotá; 2019. Disponible en: https://www.minsalud.gov.co/sites/ rid/Lists/BibliotecaDigital/RIDE/VS/ED/PSP/asiscolombia-2018.pdf

8. Así vamos en salud. Indicadores de salud. Comparativos de salud en Colombia; 2016. Disponible en: https:// www.asivamosensalud.org/indicadores/comparativosmortalidad-en-colombia/primeras-causas-demortalidad-general-en-colombia
9. Organización Mundial de la Salud (OMS). International Agency for Research on Cancer. Cancer Today. Disponible en: https://gco.iarc.fr/today/onlineanalysis-map $?_{\mathrm{v}}=2018 \&$ mode $=$ population $\&$ mode population $=$ continents \&population $=900$ \&populations $=900 \&$ key $=a s r \& \operatorname{sex}=0 \&$ can cer $=39 \&$ type $=0 \&$ statistic $=5 \&$ prevalence $=0 \&$ pop ulation_group $=0 \& a g e s \_g r o u p \% 5 \mathrm{~B} \% 5 \mathrm{D}=0 \&$ ages group $\% 5$ B $\% 5=17 \& \mathrm{nb} \_$items $=5 \&$ group cancer $=1$ \&include_nmsc $=1$ \&include_nmsc other $=1$ \&projection = natural - earth $\&$ color palette $=$ default\&map_scale $=$ quantile\&map_nb_color $\mathrm{s}=5$ \&continent $=0$ \&rotate $=\% 255 \mathrm{~B} 10 \% 252 \mathrm{C} 0 \% 255 \mathrm{D}$

10. El País. EE.UU. da luz verde al zolgensma, el medicamento más caro del mundo; 2019. Disponible en: https:/cincodias.elpais.com/cincodias/2019/05/25/ companias/1558809536_734436.html

11. Organización Mundial de la Salud (OMS). Tecnologías de asistencia; 2018. Disponible en: https://www. who.int/es/news-room/fact-sheets/detail/assistivetechnology 